\title{
Program Evaluation of Remote Heart Failure Monitoring: Healthcare Utilization Analysis in a Rural Regional Medical Center
}

\author{
William T. Riley, PhD, ${ }^{1}$ Pamela Keberlein, $R N, M S N^{2}$ \\ Gigi Sorenson, RN, MSN, ${ }^{2}$ Sailor Mohler, BS, ${ }^{3}$ Blake Tye, MPIA, ${ }^{4}$ \\ A. Susana Ramirez, $P h D^{5}$ and Mark Carroll, $M D^{2}$ \\ ${ }^{1}$ National Cancer Institute, National Institutes of Health, \\ Rockville, Maryland. \\ ${ }^{2}$ Flagstaff Medical Center, Northern Arizona Healthcare, \\ Flagstaff, Arizona. \\ ${ }^{3}$ Zephyr Technology, Annapolis, Maryland. \\ ${ }^{4}$ Qualcomm, San Diego, California. \\ ${ }^{5}$ University of California, Merced, Merced, California.
}

\begin{abstract}
Background: Remote monitoring for heart failure (HF) has had mixed and heterogeneous effects across studies, necessitating further evaluation of remote monitoring systems within specific healthcare systems and their patient populations. "Care Beyond Walls and Wires," a wireless remote monitoring program to facilitate patient and care team co-management of HF patients, served by a rural regional medical center, provided the opportunity to evaluate the effects of this program on healthcare utilization. Materials and Methods: Fifty HF patients admitted to Flagstaff Medical Center (Flagstaff, AZ) participated in the project. Many of these patients lived in underserved and rural communities, including Native American reservations. Enrolled patients received mobile, broadband-enabled remote monitoring devices. A matched cohort was identified for comparison. Results: HF patients enrolled in this program showed substantial and statistically significant reductions in healthcare utilization during the 6 months following enrollment, and these reductions were significantly greater compared with those who declined to participate but not when compared with a matched cohort. Conclusions: The findings from this project indicate that a remote HF monitoring program can be successfully implemented in a rural, underserved area. Reductions in healthcare utilization were observed among program participants, but reductions were also observed among a matched cohort, illustrating the need for rigorous assessment of the effects of HF remote monitoring programs in healthcare systems.
\end{abstract}

Key words: mobile health, telemedicine, telecardiology, home health monitoring, cardiology/cardiovascular

\section{Introduction}

A pproximately 1 million heart failure (HF) hospital admissions occur in the United States annually; ${ }^{1}$ a quarter of those are re-admitted within 30 days. $^{2}$ Healthcare costs associated with HF approach $\$ 25$ billion per year. ${ }^{3} \mathrm{HF}$ is associated with reduced survival and diminished quality of life, ${ }^{4}$ along with frequent hospitalizations and high healthcare costs. This serious public health problem is exacerbated in rural areas, which have a higher prevalence of HF and fewer treatment resources. ${ }^{5}$

Remote monitoring of HF patients has been evaluated to address these issues. A recent review documented nine meta-analyses of telemonitoring for $\mathrm{HF}$ and concluded that HF telemonitoring reduces risk of mortality, improves quality of life, and reduces healthcare costs but noted considerable heterogeneity of effects across studies. ${ }^{6}$ Well-controlled studies such as the Telemonitoring in Heart Failure (TELE-HF) $^{7}$ and Telemedical Interventional Modeling in Heart Failure $(\mathrm{TIM}-\mathrm{HF})^{8}$ failed to find an effect on mortality or hospitalizations, leading some in the field to conclude that remote monitoring $\mathrm{HF}$ is not effective. ${ }^{9}$

One potential contributor to these heterogeneous effects is the rapid change of telemonitoring technologies. The TELE-HF study, for example, required patients to call an interactive voice response system each day to record their weight, blood pressure, and symptoms. ${ }^{7}$ Using more sophisticated technologies, Seto et al. ${ }^{10}$ used a mobile phone-based remote monitoring system that wirelessly monitored weight, blood pressure, electrocardiogram, and daily symptoms and provided immediate self-management interventions to patients. Mobile and wireless technologies reduce the patient and provider burden through automated input and wireless transmission of these recordings. ${ }^{11}$

Another contributor to effect heterogeneity is the care model used for intervening upon data transmission. To be useful, telemonitoring data must be integrated in the care team workflow and with models to assist the provider on when and how to intervene with the patient based on the data provided. Wakefield et al. ${ }^{12}$ have documented a wide array of nursing interventions in response to telemonitoring data.

Differences in study samples also contribute to heterogeneity of effects across studies. Although technological advances have improved the ease of use by patients, some HF patients find remote monitoring too complex, and some are more in need of remote monitoring than others. ${ }^{13,14}$ Patients in rural areas may be more likely to benefit from remote HF monitoring than patients from urban 


\section{RILEY ET AL.}

areas, who have greater accessibility to outpatient services. ${ }^{5}$ There are sufficient differences across programs and populations to necessitate that healthcare systems engage in evaluation of the HF telemonitoring program deployed.

The purpose of the "Care Beyond Walls \& Wires" (CBWW) program was to assess the feasibility of using remote wireless monitoring via mobile broadband to facilitate patient and care team co-management of HF in a predominantly rural, disproportionately Native American patient population, but this effort also provided the opportunity to assess healthcare utilization changes resulting from this program, which is the focus of this report. The program was the result of a collaboration involving Flagstaff Medical Center (FMC) (Flagstaff, AZ), Qualcomm Wireless Reach (San Diego, CA), Zephyr Technology (Annapolis, MD), Verizon Wireless, and the National Institutes of Health. The program was implemented with patients hospitalized at FMC, a regional medical center serving a large rural area.

\section{Materials and Methods}

Fifty patients were recruited through FMC from December 12, 2011 to December 12, 2012. The FMC/Northern Arizona Healthcare Institutional Review Board reviewed and approved this project. Participants were identified primarily from HF admission diagnoses in the electronic health record, followed by project staff querying the patient's healthcare team about potential participation. As the program became known within the healthcare system, patients were referred directly by their healthcare team. In a few cases, patients were referred from outpatient care based on high care demand needs of these patients. Referrals were encouraged from patients living in rural communities and/or underserved populations; no patient was excluded based on geography, gender, race, or ethnicity.

All patients included in the program had a diagnosis of HF and were referred or cleared by their healthcare team to participate in the program. HF patients with comorbid conditions (e.g., diabetes, chronic obstructive pulmonary disease) were accepted in the program. Patients who had auditory, visual, or cognitive impairments or who were unable to speak or read English and were unable to identify a family member to assist them with the program were excluded. Patients agreed to use the program for up to 6 months and to have access to mobile broadband coverage (in home or at a nearby location that could be accessed within $24 \mathrm{~h}$ of the monitoring recording). The project team worked with patients and network providers to ensure adequate wireless network access for data transmission.

\section{MOBILE HEALTH REMOTE MONITORING INTERVENTION}

Zephyr Technology developed and provided the physiologic monitoring technology used in CBWW. The program was intended to improve transitional care coordination via remote daily monitoring to allow care teams and patients to better manage HF, provide early detection of deterioration, and provide timely interventions to avoid unnecessary hospital care. The preconfigured kit provided to each patient (pictured in Fig. 1) contained Food and Drug Administration-cleared wireless peripheral devices measuring weight, blood pressure, heart rate, and pulse oximetry. Data from these devices were transmitted to a programprovided Motorola ${ }^{\mathrm{TM}}$ (Chicago, IL) Droid X2 smartphone and either sent immediately or stored and transmitted when a wireless signal was available to the nursing care coordinator. The application aggregated the data and submitted them to their care coordinator, who gained access to the data through a clinician Web portal configured to meet Health Insurance Portability and Accountability Act requirements.

Study participants and family members were trained in the use of the kits and instructed to obtain measurements daily, review the data displayed on the Android ${ }^{\mathrm{TM}}$ (Google, Inc., Mountain View, CA) phone for accuracy and self-management purposes, and then submit the data to the nursing care coordinator via the mobile phone application.

\section{MEASURES AND PROCEDURES}

After receiving physician approval, project staff screened patients for eligibility, described the project, and obtained informed consent. Those agreeing to participate were provided HF educational information, trained in the use of the telemonitoring system, and asked to use the system daily for 3-6 months. The remotely captured health

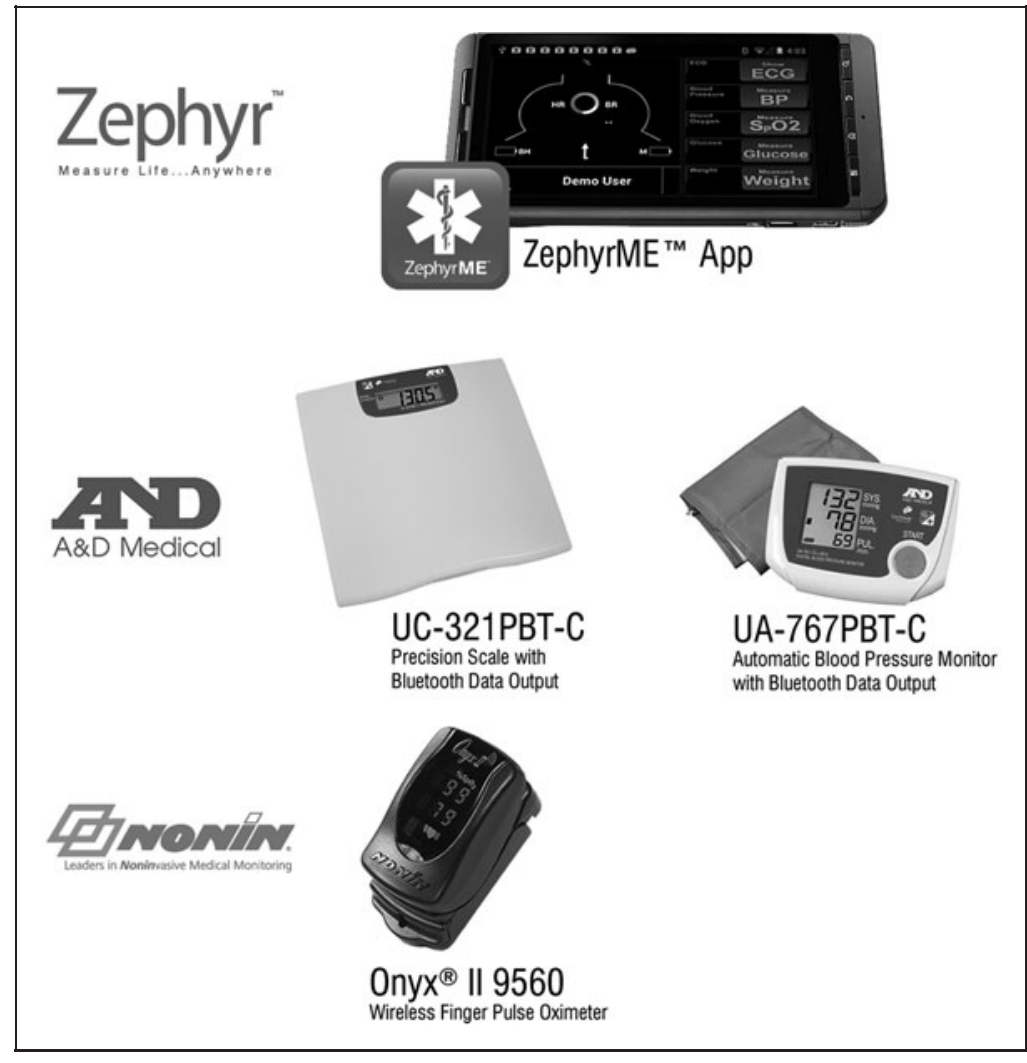

Fig. 1. "Care Beyond Walls and Wires" preconfigured kit components. 


\section{REMOTE HEART FAILURE MONITORING}

information was reviewed by FMC nursing staff, including changes in parameters over time and missing data indicative of nonadherence to monitoring. The nursing care coordination team followed up with the patients and/or family members to discuss the data, diet adherence, medication management, and other care delivery issues.

To more rigorously assess the healthcare utilization changes from the program, a matched cohort of patients was identified. A sample of those who did not participate in this remote monitoring project but were hospitalized for HF during the project period served as the pool from which to obtain a matched cohort. The control cohort was matched on gender, racial/ethnic group, age decile, severity of illness rating for the reference hospitalization, and date of hospitalization (within 7 days of the enrolled patient). When more than 1 patient matched to a given enrolled patient based on these criteria, the date closest to the enrolled patient's discharge date and the insurance status (public versus private) were used to select the most appropriate matched control. For 5 enrolled patients, an appropriate match could not be identified, and these 5 patients were excluded from analyses.

In addition to this matched cohort control, we compared patients who enrolled in the program with patients who were approached to participate but declined. Reasons provided for declining to participate included patient's difficulty in understanding the need for remote monitoring or concern that the monitoring devices would be too intrusive and/or healthcare providers declining to support their patient's participation.

For 30, 90, and 182 days prior to and following enrollment, the medical records of the enrolled, declined, and matched control patients were queried to collect the number of inpatient and emergency department (ED) admissions, the number of days of inpatient and ED admissions (ED admissions coded as 1 day), and the total FMC charges for these admissions. For the matched cohort, the date of enrollment of the matched enrolled participant was the date of "enrollment" for the matched cohort. Although FMC is not a closed system, it is the predominant healthcare system serving this community, and the likelihood of inpatient healthcare services being provided outside of the FMC system is small; therefore, these charges are a reasonable estimate of inpatient and ED healthcare utilization.

\section{STATISTICAL ANALYSES}

Discrete data were analyzed via chi-square tests, and continuous data were analyzed via paired $t$ tests. Primary analyses were repeatedmeasures analyses of variance comparing enrolled versus declined and enrolled versus matched control groups at 30, 90, and 180 days prior to and following enrollment on the three health utilization indices: number of re-admissions after enrollment, days of hospitalization, and hospital charges. Because these health indices are positively skewed with zero values, these repeated-measures analyses of variance were also performed on the $\log$ transformations $([\log 10(x+1)]$ of these values. $F$ statistics reported are based on the log-transformed data, but the untransformed means and standard deviations are reported to facilitate interpretation. As exploratory analyses of a small program evaluation, multiple comparisons were performed without adjustments for alpha inflation (significance at $p<0.05$ ).

\section{Results}

\section{SAMPLE DESCRIPTION}

Demographic data by group (enrolled, matched, declined) are presented in Table 1. The matching procedures resulted in enrolled and matched groups with nearly identical sociodemographics. Compared with the enrolled group, those who declined to participate were more likely to be male and Native American, but these differences were not statistically significant. Severity of illness ratings also did not differ significantly among groups $\left[\chi^{2}(6)=9.89, p=0.13\right]$.

\section{HEALTHCARE UTILIZATION OF ENROLLED GROUP PRE- TO POST-INTERVENTION}

Tables 2-4 show the number of hospitalizations, number of days of hospitalization, and the hospital charges, respectively, for the cumulative 30, 90, and 182 days before and after enrollment. For all three health utilization indices across all three time periods, there was a significant $(p<0.05)$ reduction in healthcare utilization based on paired-sample $t$ tests. For example, for the 6 months prior to versus following program enrollment, the average number of hospitalizations decreased $42 \%$, from 3.3 to 1.9 admissions, the average number of days hospitalized decreased 64\%, from 14.2 to 5.2 days, and the average total charges decreased $67 \%$, from $\$ 138,600$ to $\$ 44,673$. Comparably significant reductions were found for the 30- and 90-day periods prior to versus following enrollment.

There were five known deaths in the enrolled group that occurred between the 90- and 182-day follow-up, and the 6-month analyses were repeated excluding those who died. Among those who survived to 6 months post-enrollment, the average number of hospitalizations decreased $44 \%$, from 3.0 to 1.7 [ $t(39)=2.2, p=0.035$ ], average total number of days hospitalized decreased 64\%, from 13.2 to 4.8 $[t(39)=5.95, p<0.0001]$, and average total charges decreased $72 \%$, from $\$ 129,480$ to $\$ 36,914$ [ $t(39)=5.6, p<0.0001]$.

\begin{tabular}{|c|c|c|c|}
\hline & ENROLLED & MATCHED & DECLINED \\
\hline Number of subjects & 45 & 45 & 57 \\
\hline $\begin{array}{l}\text { Mean (SD) age } \\
\text { (years) }\end{array}$ & $66.0(14.5)$ & $65.9(14.7)$ & $66.35(14.1)$ \\
\hline Percentage female & $48.9 \%$ & $48.9 \%$ & $36.8 \%$ \\
\hline Percentage white & $51.1 \%$ & $51.1 \%$ & $50.9 \%$ \\
\hline $\begin{array}{l}\text { Percentage Native } \\
\text { American }\end{array}$ & $31.1 \%$ & $31.1 \%$ & $36.8 \%$ \\
\hline $\begin{array}{l}\text { Percentage } \\
\text { Hispanic }\end{array}$ & $15.6 \%$ & $15.6 \%$ & $10.5 \%$ \\
\hline
\end{tabular}

$\mathrm{SD}$, standard deviation. 
Table 2. Number of Hospitalizations Within 30, 90, and 182 Days Prior to and Following Enrollment for the Enrolled, Declined, and Matched Cohort Groups

\begin{tabular}{|l|l|c|} 
& & 182 DAYS \\
30 DAYS & 90 DAYS & (6 MONTHS)
\end{tabular}

Enrolled $^{a}$

\begin{tabular}{l|l|l|l}
\hline Pre & $1.11(1.07)$ & $2.20(2.17)$ & $3.31(3.31)$ \\
\hline Post & $0.47(1.10)$ & $1.24(3.24)$ & $1.87(4.54)$ \\
\hline Declined & $0.70(0.84)$ & $1.33(1.57)$ & $1.86(3.03)$ \\
\hline Pre & $0.44(0.68)$ & $0.93(1.24)$ & $1.49(2.01)$ \\
\hline Post & $1.09(0.92)$ & $1.98(2.12)$ & $2.42(2.99)$ \\
\hline Matched & $0.56(0.87)$ & $0.87(1.44)$ & $1.22(1.71)$ \\
\hline Pre &
\end{tabular}

Data are mean (standard deviation) values.

${ }^{a}$ For enrolled paired, $t(44)=5.39, p<0.0001$ for 30 days; $t(44)=2.72, p=0.009$ at 90 days; and $t(44)=2.56, p=0.14$ at 182 days. For enrolled versus declined, at 30 days, time $F(1,100)=25.09, p<0.0001$, time $\times$ group $=5.13, p=0.026$; at 90 days, time $F(1,100)=21.98, p<0.0001$, time $\times$ group $=6.04, p=0.016$; and at 182 days, time $F(1,100)=16.61, p<0.0001$, time $\times$ group $=8.67, p=0.004$. For enrolled versus matched, at 30 days, time $F(1,88)=43.87, p<0.0001$, time $\times$ group $=0.63$, $p=0.429$; at 90 days, time $F(1,88)=50.87, p<0.0001$, time $\times$ group $=0.12$, $p=0.727$; and at 182 days, time $F(1,88)=45.36, p<0.0001$, time $\times$ group $=1.00$, $p=0.320$

\section{ENROLLED VERSUS DECLINED ON HEALTHCARE UTILIZATION CHANGES}

For all three healthcare utilization indices and all three time frames, a significant main effect for time and a significant time by group interaction were found for the enrolled versus declined group. As shown in Tables 2-4, these significant findings were in the direction of greater reductions in healthcare utilization for the enrolled group versus the declined group. For example, for the 6 months prior to versus following enrollment, the average number of hospitalizations decreased 43\%, from 3.3 to 1.9, in the enrolled group and 20\%, from 1.9 to 1.5 , in the declined group. Average number of days hospitalized decreased 63\%, from 14.2 to 5.2 , in the enrolled group and 13\%, from 8.1 to 7.1, in the declined group. Average hospital charges decreased $67 \%$, from $\$ 138,600$ to $\$ 44,674$, among the enrolled and 3\%, from $\$ 75,549$ to $\$ 73,593$, among the declined. Across utilization indices and time frames, the declined group had lower preenrollment utilization than the enrolled group.

\section{ENROLLED VERSUS MATCHED CONTROLS ON HEALTHCARE UTILIZATION CHANGES}

Tables 2-4 show the healthcare utilization reductions for enrolled versus matched controls across time frames (30, 90, and 182 days) and healthcare utilization indices. All main effects for time were significant, but none of the time by group interactions was signifi-
Table 3. Total Days of Hospitalization Within 30, 90, and 182 Days Prior to and Following Enrollment for the Enrolled, Declined, and Matched Cohort Groups

for

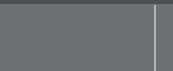

30 DAYS

182 DAYS

Enrolled $^{a}$

\begin{tabular}{l|c|c|c}
\hline Pre & $5.09(4.69)$ & $9.42(7.33)$ & $14.22(12.36)$ \\
\hline Post & $1.31(2.57)$ & $3.02(5.21)$ & $5.24(8.42)$ \\
\hline Declined & \multicolumn{5}{|l}{} \\
\hline Pre & $4.28(6.66)$ & $7.12(8.48)$ & $8.11(9.57)$ \\
\hline Post & $2.28(5.31)$ & $4.79(6.48)$ & $7.05(9.23)$ \\
\hline Matched & $4.80(5.51)$ & $7.24(6.58)$ & $8.36(8.20)$ \\
\hline Pre & $1.44(2.19)$ & $3.27(7.89)$ & $4.60(9.19)$ \\
\hline Post &
\end{tabular}

Data are mean (standard deviation) values.

${ }^{a}$ For enrolled paired, $t(44)=5.22, p<0.0001$ for 30 days; $t(44)=4.87, p<0.0001$ for 90 days; and $t(44)=5.98, p<0.0001$ for 182 days. For enrolled versus declined, at 30 days, time $F(1,100)=26.17, p<0.0001$, time $\times$ group $=3.81, p=0.054$; at 90 days, time $F(1,100)=24.04, p<0.0001$, time $\times$ group $=7.04, p=0.009$; and at 182 days, time $F(1,100)=18.54, p<0.0001$, time $\times$ group $=9.85, p=0.002$. For enrolled versus matched, at 30 days, time $F(1,88)=48.09, p<0.0001$, time $\times$ group $=0.819$, $p=0.368$; at 90 days, time $F(1,88)=59.09, p<0.0001$, time $\times$ group $=0.093$, $p=0.338$; and at 182 days, time $F(1,88)=50.94, p<0.0001$, time $\times$ group $=1.62$, $p=0.207$.

cant. For the 6 months prior to versus following enrollment, there were two significant main effects for group for the days of hospitalization $[F(1,100)=5.05, p=0.027]$ and for hospital charges $[F(1,100)=5.03, p=0.027]$ in the direction of lower utilization in the matched control compared with the enrolled group over time. No other significant main effects for group were found. As shown in Tables 2-4, the reductions in healthcare utilization among the matched controls were comparable to the reductions among the enrolled.

\section{REHOSPITALIZATION RATES}

Because these healthcare utilization indices are positively skewed, we performed two additional sets of analyses. First, we compared the groups on rehospitalization rates for 30,90 , or 182 days. These are the total number of hospitalizations during these time periods, not the more narrow Centers for Medicare \& Medicaid Services-defined definition of HF rehospitalization. ${ }^{15}$ At 30 days, 33\% of enrolled, $35 \%$ of declined, and $40 \%$ of matched controls had been hospitalized one or more times. At 90 days, $47 \%$ of enrolled, $54 \%$ of declined, and $44 \%$ of matched controls had been hospitalized one or more times. At 6 months, $64 \%$ of enrolled, $60 \%$ of declined, and $51 \%$ of matched controls had been hospitalized one or more times. Rehospitalization rates were not significantly different between groups.

Log transformations of the number of hospitalizations, days hospitalized, and charges were also computed to normalize these data. 


\section{REMOTE HEART FAILURE MONITORING}

Table 4. Total Hospital Charges (Dollars) Within 30, 90, and 182 Days Prior to and Following Enrollment

for the Enrolled, Declined, and Matched Cohort Groups

182 DAYS

30 DAYS 90 DAYS $\quad$ (6 MONTHS)

\begin{tabular}{c|c|c|c} 
Enrolled $^{\mathrm{a}}$ & \multicolumn{4}{c}{} \\
\hline Pre & $50,890(55,737)$ & $88,554(86,469)$ & $138,600(136,741)$ \\
\hline Post & $11,467(23,488)$ & $26,297(48,780)$ & $44,674(717,509)$ \\
\hline Declined \\
\hline Pre & $40,919(69,497)$ & $71,247(86,469)$ & $75,549(86,091)$ \\
\hline Post & $27,905(77,198)$ & $48,719(84,163)$ & $73,593(107,725)$ \\
\hline Matched & \multicolumn{5}{|c}{} \\
\hline Pre & $53,113(72,869)$ & $73,382(78,435)$ & $92,930(102,947)$ \\
\hline Post & $11,542(19,242)$ & $26,935(56,578)$ & $39,732(71,750)$ \\
\hline
\end{tabular}

Data are mean (standard deviation) values.

${ }^{a}$ For enrolled paired, $t(44)=4.57, p<0.0001$ for 30 days; $t(44)=4.26, p<0.0001$ for 90 days; and $t(44)=5.53, p<0.0001$ for 182 days. For enrolled versus declined, at 30 days, time $F(1,100)=24.63, p<0.0001$, time $\times$ group $=4.73, p=0.032$; at 90 days, time $F(1,100)=20.33, p<0.0001$, time $\times$ group $=4.95, p=0.028$; and at 182 days, time $F(1,100)=11.29, p<0.0001$, time $\times$ group $=3.68, p=0.058$. For enrolled versus matched, at 30 days, time $F(1,88)=41.29, p<0.0001$, time $\times$ group $=0.87$, $p=0.352$; at 90 days, time $F(1,88)=47.29, p<0.0001$, time $\times$ group $=0.15$, $p=0.698$; and at 182 days, time $F(1,88)=32.78, p<0.0001$, time $\times$ group $=0.02$, $p=0.891$.

The repeated-measures analysis of variance $F$ statistics shown in Tables 2-4 are based on these log-transformed data. Comparable repeated-measures analysis of variance findings were obtained using the untransformed data.

\section{SATISFACTION AND USABILITY RATINGS AMONG ENROLLED}

After the 6-month monitoring period, participants were asked to provide satisfaction and usability ratings. Only 14 patients provided these ratings. On a 5-point scale, these patients reported ratings of 4 or 5 for all of the satisfaction and usability ratings with the exception of the equipment being easy to use and the overall experience, for which 1 patient rated these 2 and 3, respectively.

\section{Discussion}

The CBWW program provided the opportunity to assess the effects of a mobile health remote monitoring program for HF patients on hospital healthcare utilization in a rural and disproportionately $\mathrm{Na}-$ tive American population. Thirty-one percent of participants were Native American, and the county primarily served by FMC (Coconino County, Arizona) has a population density of 7.2 compared with 87.4 people per square mile in the United States. ${ }^{16}$ The healthcare utilization of program participants was evaluated by comparing the number of hospitalizations, days hospitalized, and hospital charges in the 6 months prior to and following enrollment, both within the enrolled participants group and compared with those who declined to participate and to a matched cohort. The results from this evaluation were mixed. The HF patients enrolled in this project reduced their healthcare utilization within the healthcare system by over half from the periods prior to versus following enrollment. Even when those who had died were excluded, the remaining participants had a $72 \%$ reduction in hospital charges from the 6 months prior to versus following enrollment (from $\$ 129,480$ to $\$ 36,914$ ).

Compared with the group of patients who declined to participate in this pilot program, the enrolled group showed greater reductions in healthcare utilization across all measured indices and time frames. Although the declined group had much smaller reductions in healthcare utilization than the enrolled group, the declined group also appeared to have lower healthcare utilization prior to enrollment compared with the enrolled group. High rates of hospitalization in the months preceding this remote monitoring program may have motivated the enrolled patients and/or their healthcare providers to participate in the program.

To address the confounds of comparing enrolled versus declined patients, we identified a matched cohort control group for comparison. The enrolled and matched control groups showed similar reductions in healthcare utilization. The reductions observed in the matched control group suggest that the various HF management programs that were concurrently implemented in this healthcare system potentially had an impact in reducing re-admissions and that for a remote monitoring system to be shown effective in a subsequent controlled trial, it must produce a separate and quantifiable additive effect over and above existing HF management programs. For this small program evaluation compared with matched controls, we failed to find a significant effect of the CBWW program on healthcare utilization.

Although we were able to match the enrolled patients on key demographic variables and dates of hospitalizations, a range of unknown variables such as type or severity of HF condition, comorbidities, and/or the presence of supportive home environment could have differed between the enrolled and matched controls. Mortality status was unknown in the matched control group so it is possible that the reduction in healthcare utilization in this group was the result of higher mortality rates in the matched control compared with the enrolled groups.

Random assignment to a control condition is preferable but often not possible when these remote monitoring technologies are considered by hospitals. This project demonstrates these new remote monitoring technologies can be reasonably evaluated as they are being piloted and tested by healthcare systems. It also provides a cautionary note about concluding that observed reductions in healthcare utilization are due to the introduction of these remote monitoring programs when other factors could be responsible for these reductions. Given that this is a limited evaluation of a small pilot program, it would also be premature to conclude that the remote monitoring program could not produce a significant additive effect in a larger, well-controlled study with planned end points, but this evaluation failed to find such an effect. 


\section{RILEY ET AL.}

Remote monitoring for HF has an intuitive appeal as a strategy to reduce re-admissions and better manage these patients. It is important to recognize that this project was only able to assess hospitalbased healthcare utilization, not all healthcare utilization, and not mortality or quality of life, which in combination would provide a more complete understanding of the impact of remote monitoring systems on HF patients. Healthcare providers also may need more experience and training with these systems and the data they provide to better manage their patients based on these data. Much more must be learned about the effects of remote monitoring for HF, not only on patients but also on their caregivers and healthcare providers, to optimize the management of HF patients.

\section{Acknowledgments}

We thank Barbara Mittleman of the National Institutes of Health Public-Private Partnership office for initiating the partnership that resulted in this project and, from Qualcomm, Michal Koenig for technical support and Robert Jarrin for implementation guidance. We also thank the many staff of FMC, especially Kelly DeGraff, who worked to facilitate the adoption of this program and coordinated the care of patients in the program. This project was supported by Qualcomm Wireless Reach, and Zephyr Technology provided in-kind support for the telemonitoring system used in this project.

\section{Disclosure Statement}

S.M. is an employee of Zephyr Technology. B.T. is an employee of Qualcomm. W.T.R., P.K., G.S., A.S.R., and M.C. declare no competing financial interests exist.

\section{REFERENCES}

1. Ross JS, Chen J, Lin Z, Bueno H, Curtis JP, Keenan PS, et al. Recent national trends in readmission rates after heart failure hospitalization. Circ Heart Fail 2010;3:97-103.

2. Jencks SF, Williams MV, Coleman, EA. Rehospitalizations among patients in the Medicare fee-for-service program. N Engl J Med 2009;360:1418-1428.

3. Roger VL, Go AS, Lloyd-Jones DM, et al. Heart disease and stroke statistics2012 update: A report from the American Heart Association. Circulation 2012;125:e2-e220.

4. Roger VL. Epidemiology of heart failure. Circ Res 2013;113:646-659.

5. Clark RA, Eckert KA, Stewart S, Phillips SM, Yallop JJ, Tonkin AM, Krum H. Rural and urban differentials in primary care management of chronic heart failure: New data from the CASE study. Med J Aust 2007;186:441-445.
6. Purcell $R$, Mclnnes $S$, Halcomb EJ. Telemonitoring can assist in managing cardiovascular disease in primary care: A systematic review of systematic reviews. BMC Fam Pract 2014;15:43-56.

7. Chaudhry SI, Mattera JA, Curtis JP, Spertus JA, Herrin J, Lin Z, et al. Telemonitoring in patients with heart failure. N Engl J Med 2010;363: 2301-2309

8. Koehler F, Winkler S, Schieber M, Sechtem U, Stangl K, Bohm M, et al. Impact of remote telemedical management on mortality and hospitalizations in ambulatory patients with chronic heart failure: The Telemedical Interventional Monitoring in Heart Failure Study. Circulation 2011;123:1873-1880.

9. Abraham WT. Remote heart failure monitoring. Curr Treat Options Cardiovasc Med 2013;15:556-564.

10. Seto E, Leonard KJ, Cafazzo JA, Barnsley J, Masino C, Ross HJ. Mobile phonebased telemonitoring for heart failure management: A randomized controlled trial. J Med Internet Res 2012;14:e31.

11. Anand IS, Greenberg BH, Fogoros RN, Libbus I, Katra RP; MUSIC Investigators. Design of the Multi-sensor Monitoring in Congestive Heart Failure (MUSIC) study: Prospective trial to assess the utility of continuous wireless physiological monitoring in heart failure. J Card Fail 2011;17:11-16.

12. Wakefield BJ, Scherubel M, Ray A, Holman MA. Nursing interventions in a telemonitoring program. Telemed J E Health 2012;19:160-165.

13. Boyne JJJ, Vrijhoef HJM. Implementing telemonitoring in heart failure care: Barriers from the perspectives of patients, healthcare professionals, and healthcare organizations. Curr Heart Fail Rep 2013;10:254-261.

14. Seto E, Leonard KJ, Cafazzo JA, Barnsley J, Masino C, Ross HJ. Perceptions and experiences of heart failure patients and clinicians on the use of mobile phonebased telemonitoring. J Med Internet Res 2012;14:e25.

15. Centers for Medicare \& Medicaid Services. Readmissions reduction program. Available at www.cms.gov/Medicare/Medicare-Fee-for-Service-Payment/ AcutelnpatientPPS/Readmissions-Reduction-Program.html (last accessed July 10, 2014).

16. U.S. Census Bureau. 2010 Census of Population and Housing, Population and Housing Unit Counts, CPH-2-4, Arizona. Washington, DC: U.S. Government Printing Office, 2012. Available at https://www.census.gov/prod/cen2010/ cph-2-4.pdf (last accessed July 10, 2014).

Address correspondence to: William T. Riley, PhD

National Cancer Institute 9609 Medical Center Drive, MSC 9761 Rockville, MD 20850

E-mail: wiriley@mail.nih.gov

Received: May 12, 2014

Accepted: May 30, 2014 